\title{
Diferenças de gênero no estilo de atribuições causais e metas acadêmicas de
}

\section{universitários brasileiros}

\author{
Gender differences in the style of causal attributions and academic goals of \\ Brazilian University
}

Suely Aparecida do N. Mascarenhas*, Juan Carlos Brenlla, Alfonso Barca**

*Universidade Federal do Amazonas, Humaitá, Brasil, ** Universidade da Coruña, España

\begin{abstract}
Resumo
As teorias das atribuições causais e a das metas acadêmicas têm motivado diversos estudos e pesquisas associados ao tema em diferentes contextos históricos e geográfico do planeta. A pesquisa foi realizada ao abrigo de projeto apoiado pelo $\mathrm{CNPq}$ com apoio de equipe multidisciplinar junto a duas universidades federais do Brasil (UFAM e UNIR) contou com $\mathrm{n}=1355$ participantes do sexo masculino e feminino. Os resultados demonstram as metas visando a valorização e necessidade de se sobressair. Quanto às atribuições causais para o rendimento, foi observada diferenças favoráveis aos estudantes do sexo masculino $\mathrm{p} \leq .001$, confirmando resultados de pesquisas na área.
\end{abstract}

Palavras chave: atribuições causais, metas acadêmicas, ensino superior, diferenças de gênero.

Segundo a Teoria das Atribuições causais, os acontecimento $\mathrm{s}$ externos não determinam diretamente as reações dos indivíduos ( comportamento e emoções), sendo essas ligações S-R mediadas pelas atribuições causais que são as explicações pessoais e subjetivas que as pessoas dão aos fatos que vivenciam. Nesta perspectiva, os indivíduos estão geralmente motivados para atingir uma compreensão realista das causas que produziram os diversos acontecimentos no seu contexto pessoal. Essa compreensão causal tem como função alcançar determinados objetivos pessoais, adquirir a mestria cognitiva e assegurar a sobrevivência do indivíduo (Oliveira, 1996).

A teoria das atribuições causais vem sendo amplamente estudada nos últimos 20 anos, fornecendo assim, instrumentos conceituais para a exploração de fenômenos psicológicos em diversos âmbitos, tais como a realização escolar, o relacionamento interpessoal, os comportamentos de ajuda, a psicologia organizacional, a motivação para o poder e a psicopatologia, dentre outros (Weiner, 1985; 2004).

A teoria das atribuições causais surgiu do estudo das motivações humanas, de acordo com Weiner (2004), a psicologia apresenta a preocupação com essa temática e a define como um estado interior que estimula, direciona e mantêm um comportamento. Sales (2010) aponta que pode-se adotar uma definição geral e coerente a partir da teoria cognitiva, que conceitua a motivação como um processo por meio do qual uma atividade é direcionada, instigada e sustentada. A teoria da atribuição causal, utilizada nesse trabalho, pressupõe que as pessoas podem atribuir causas ou motivos às suas ações (Weiner, 1985; Beck 2001; Mascarenhas, 2004; Mascarenhas, Almeida \& Barca, 2005; Almeida \& Guisande, 2010; Sales, 2010).

Weiner (1985) enfoca que há uma relação entre a atribuição de causalidade com o sucesso e o fracasso na realização de tarefa, de forma que crenças e expectativas depositadas nessa realização podem favorecer um desses aspectos. Além disso, defende que a autoestima, o autoconceito e as expectativas são grandes determinantes do desempenho em tarefas e são influenciados por aquilo que o indivíduo atribui como causa do sucesso ou do fracasso.

Para classificar as causas atribuídas ao desempenho positivo ou negativo em situações de realização, Weiner (1985), elaborou uma taxonomia na qual a causa apresenta três dimensões, sendo elas: o Lócus da Causalidade, a Estabilidade e a Controlabilidade.

$\mathrm{Na}$ dimensão do Lócus da Causalidade as causas são distribuídas a fatores internos ou externos ao indivíduo e nela estariam incluídas causas como o esforço típico, esforço imediato, habilidade, humor, fadiga e doença, como causas internas; estilo de ensino do professor, dificuldade da tarefa, sorte e ajuda não usual de outros, como causas externas.

Visualizar as atribuições causais pode remeter ao componente emocional e afetivo da aprendizagem escolar; o que é realizado porque os estudantes estabelecem distinção entre o que aprendem de maneira efetiva ou o que não aprendem; demonstrando que sabem atribuir uma causa para a sua aprendizagem a partir de conceitos pessoais, o que influencia diretamente na autoestima, no autoconceito e no rendimento acadêmico/desempenho escolar (Silva, Mascarenhas \& Silva, 2011),

Quanto às metas acadêmicas destacamos que dentre os modelos teóricos abordados mundialmente na investigação da motivação no contexto escolar, destacase a teoria de metas de realização que tem trazido grandes contribuições para o entendimento dos fatores 
motivacionais que influenciam o comportamento dos estudantes, pois busca explicar a motivação focalizando o aspecto qualitativo do envolvimento no processo individual de aprendizagem.

O sentido conceitual de meta refere-se ao aspecto qualitativo do envolvimento do aluno em situações de aprendizagem, expressando em diferentes níveis o propósito, ou o porquê, de uma pessoa envolver-se em uma tarefa, ou seja, a meta de realização que a pessoa adota representa o motivo ou a razão pela qual ela irá realizar determinada tarefa.

As metas de realização vêm sendo amplamente estudadas, bem como as suas relações com os demais construtos motivacionais. As metas exprimem as razões das escolhas do indivíduo.

$\mathrm{Na}$ literatura consultada sobre a motivação, o resultado das metas acadêmicas, converteu-se em uma das principais linhas de investigação no campo da motivação acadêmica, dada sua relevância sobre a cognição, o comportamento e o afeto. Ames (1990) definiu as "metas de realização" como um conjunto de pensamentos, crenças, propósitos e emoções que traduzem as expectativas dos alunos em relação a determinadas tarefas que deverão executar, ou seja, as metas são representadas por modos diferentes de enfrentar as tarefas acadêmicas.

Para Mascarenhas (2004), metas acadêmicas são multidimensionais com causas não excludentes, pelas quais os estudantes realizam suas atividades de estudo (agradar pais e familiares, ser reconhecidos pelos amigos, conseguir um bom emprego, desenvolver-se como pessoa).

Uma meta acadêmica pode ser considerada como um modelo integrado de motivação, ou o estilo de crenças, atribuições e emoções/sentimentos que impulsionam as intenções comportamentais, o que os sujeitos fazem, ou querem fazer, no contexto educacional de ensino/aprendizagem. Esses objetivos poderão determinar as reações afetivas do sujeito cognitivo e comportamental para o resultado de sucesso ou de fracasso, como a quantidade e a qualidade de suas atividades de aprendizagem e de estudo (Mascarenhas, 2004).

Dentro desta tradição, as metas são conceituadas com o propósito, ou o núcleo dinâmico cognitivo de compromisso, com a tarefa e o tipo de meta adotado, estabelecendo o marco geral mediante o qual os indivíduos interpretam e experimentam os contextos de resultados.

Esta construção teórica de Metas trabalha com o conceito de motivação para realização, que contempla a relação entre motivação e metas, essas que por sua vez afetam os modos pelos quais as pessoas abordam as tarefas, ou seja, seu comportamento de realização .

Inicialmente, três tipos de metas de realização foram propostas: i) metas de aprendizagem relacionadas com uma orientação voltada para o envolvimento com a aprendizagem, o conhecimento e o desenvolvimento de competências de mestria; (ii) metas orientadas para a performance ou desempenho, em que sujeito procura sobretudo obter bons desempenhos ou resultados para, por exemplo, avançar nos seus estudos; e, (iii) metas orientadas para a obtenção da consideração positiva, ou aprovação, por parte dos outros significativos (pais, professores ou colegas) e evitar a rejeição e julgamentos desfavoráveis da sua competência (Mascarenhas, Boruchovitch, Maciel \& Silva 2013; Mascarenhas, Silva, Silva, Morais, Aguiar, Peluso \& Barca, 2013; Mascarenhas \& Morais 2013; Miras, 2004; Morais \& Mascarenhas, 2012) .

Os estudantes com orientação à meta aprender entendem que o sucesso nas realizações escolares consiste na melhora em conhecimentos e habilidades, em progredir, dominar sempre mais os conteúdos, com inovação e criatividade.

Nessa perspectiva, podemos dizer que o aluno orientado à meta aprender caracteriza-se como aquele que busca o crescimento intelectual, que valoriza o esforço pessoal, que enfrenta os desafios e costuma utilizar-se de estratégias de aprendizagem mais efetivas e profundas (Rodrigues, 1984; Sales,2010; Silva, Mascarenhas \& Silva 2011; Weiner, 2004.

\section{Participantes}

\section{MÉTODO}

A pesquisa foi realizada ao abrigo de projeto apoiado pelo CNPq com apoio de equipe multidisciplinar junto a duas universidades federais do Brasil (UFAM e UNIR) contou com $n=1355$ participantes do sexo masculino e feminino que responderam a instrumento próprio, observando procedimentos éticos vigentes, tendo obtido aprovação do Comitê de Ética em Pesquisa da UFAM.

\section{Instrumento}

$\mathrm{O}$ instrumento utilizado para avaliar as atribuições de causalidade para o rendimento acadêmico foi a escala QEAP 44, organizada em escala likert de 5 pontos 1 . Totalmente em desacordo e 5. Totalmente de acordo (Barca \& Porto, 2005), adaptada por Mascarenhas, 2008.

\section{Procedimentos de coleta, tratamento e análise de dados}

A Escala QEAP-44 foi aplicada por equipe de colaboradores orientados pela pesquisadora responsável nas unidades da UFAM e UNIR. Os estudantes responderam voluntária e anonimamente ao instrumento observando procedimentos éticos internacionais, de modo individual em horário de aula previamente agendado com os professores após serem informados dos objetivos da pesquisa. O tempo de resposta variou entre 5 e 15 minutos, $\underline{M}=10 \mathrm{~min}$

Após a coleta dos dados os mesmos foram enviados ao LAPESAM, Unidade da UFAM, IEAA, Humaitá via malote ou correios sedex onde após a conferência e organização receberam tratamento estatístico com apoio do programa SPSS de acordo com os objetivos da pesquisa. Foram realizadas diversas análises estatísticas, todavia pelas características deste trabalho, registramos diferenças de gênero no que se refere aos constructos em estudo. 


\section{RESULTADO E DISCUSSÃO}

Os resultados demonstram indicadores semelhantes aos encontrados na literatura especializada realizadas em outros cenários acadêmicos (Aguiar \& Mascarenhas, 2010; Aguiar, Mascarenhas \& Barca, 2010; Almeida \& Guisande, 2010; Ames, 1990; Barca, 1999ab; Barca, 2000; Barca \& Peralbo, 2002; Barca, 2009; Brenlla, 2005; Beck, 2010; Faria, 2010; Figueira \& Lobo, 2010; León \& Delgado, 1998;Mascarenhas, 2004 ; b; Mascarenhas, Almeida \& Barca, 2005; Mascarenhas, Barca, Silva, Macie 1\& Silva, 2013).

Da análise dos resultados aportados, constata-se que no cenário do ensino superior público onde a pesquisa foi realizada (Amazonas - UFAM e Rondônia - UNIR), predomina metas acadêmicas de valorização externa entre os estudantes universitários:

(i) "Normalmente me esforço em meus estudos porque quero ser valorizado/a por meus amigos e companheiros de classe", $\mathrm{p} \leq .001$;

(ii) "Esforço-me em meus estudos porque quero obter as melhores notas da turma" $\mathrm{p} \leq .001$;

(iii) "Esforço-me em meus estudos porque quero me sobressair" $\mathrm{p} \leq .001$.

No que se refere às atribuições causais para o rendimento, foi observada diferença no item "As boas notas que obtenho se devem sempre à minha capacidade" $\mathrm{p} \leq .001$.

Todas as diferenças favoráveis aos estudantes do sexo masculino $\mathrm{p} \leq .001$, confirmando resultados de pesquisas na área.

Quanto à validade do instrumento, o estudo revelou sua validade estatística com o indicador Alpha de Cronbach superior a ,70.

\section{CONCLUSÃO}

Da análise das informações aportadas pela pesquisa ficou demonstrado o estilo motivacional que busca recompensas ou valorização externa.

Por outro lado, a pesquisa evidenciou diferença significativa positiva para os estudantes do sexo masculino no que se refere ao autoconceito acadêmico, confirmando resultados de outras pesquisas na área.

A continuidade de pesquisas e estudos na área em diferentes contextos sociais e educativos poderá contribuir, a exemplo da pesquisa em pauta, com o aporte de novas informações sistematizadas que possam apoiar a proposição de políticas públicas que considerem as diferenças de gênero no ensino superior.

\section{Agradecimentos:}

Os autores agradecem o apoio financeiro do CNPq.

\section{REFERÊNCIAS}

Aguiar, R. S.; Mascarenhas, S. A. N. (2010). Avaliação dos enfoques de aprendizagem, atribuições causais, representações sociais, relações família universidade, bem-estar subjetivo e rendimento de universitários do Amazonas/UFAM. Relatório técnico de iniciação científica-PIB-
H005/UFAM/FAPEAM. Atas do II Congresso de Iniciação Científica do Campus Vale do Rio Madeira, ISSN - 1984-9850. Humaitá-AM.

Aguiar, R. S.; Mascarenhas, S. A. N.; Fernandes, F. S.; Barca, (2010). A. Diferenças de gênero verificadas a partir da escala QEAP-44 quanto ao estilo de atribuições causais para a aprendizagem: uma investigação com estudantes do ensino superior dos municípios de Humaitá e Apuí Amazonas/Brasil. In: Actas do I Seminário Internacional "Contributos da Psicologia em Contextos Educativos". Braga: Universidade do Minho, ISBN- 978-972-8746-87-2.

Almeida, L. S.; Guisande, M. A. (2010). Atribuições causais na explicação da aprendizagem escolar. In: Boruchovitch, E.; Bzuneck, J. A.; Guimarães, S. E. R. (Orgs). Motivação para aprender: aplicações no contexto educativo. Petrópolis: Vozes.

Ames, C. (1990). Motivation: what teacher ned to know. Teacher college record, 91, 409-421.

Barca, A. (2000). Escala SIACEPA: Sistema Integrado de Evaluación de Atribuciones Causales y Procesos de Aprendizaje (Educación Secundaria). A Coruña: Publicaciones de la Revista GalegoPortuguesa de Psicoloxía e Educación. Universidade da Coruña/Universidade do Minho.

Barca, A. Y Peralbo, M.(2002). Informe Final del Proyecto FEDER/ESOG-Galicia (1FD97-0283). Los contextos de aprendizaje y desarrollo en la Educación Secundaria Obligatoria (ESO): Perspectivas de intervención psicoeducativa sobre el Fracaso escolar en la Comunidad Autónoma de Galicia. Madrid: Dirección General de Investigación (I+D). Ministerio de Ciencia y Tecnología.

Barca, A. (Coord.) (2009). Motivacíon y aprendizaje em contextos educativos. Granada: Grupo Editorial Universitário.

Barca, A. \& Porto, A. (2005). Escala QEAP 44, Universidade da Corunhã, A Corunha, Espanha.

Brenlla, J. C. (2005). Atribuciones causales, enfoques de aprendizaje, rendimiento académico y compretencias bilingües en alumnos de educación secundaria. Un análisis multivariable. A Coruña: Universidad de A Coruña (tesis doctoral, inédita).

Beck, M. L. G. (2001). A teoria da atribuição causal e sua relação com a educação. In: Rev. Maringá/PR - ano I - n.3, quadrimestral, dezembro: 2001. Disponível em: <http://www.urutagua.uem. br//03Beck.htm> Recuperado em: 09 abril 2010.

Faria, L. (2010).Aspectos desenvolvimentais das atribuições e dimensões causais: estudos no contexto português. In: RIDEP, v.9, n1, año 2000. Disponível em: < http://www. aidep. org/03 ridep /R09/ R092.pdf $>$ Recuperado em 09 abril 2010.

Figueira, A. P. C.; Lobo, R. A. C. T. (2010).Academic failure: causal attributions, personal conceptions of intelligence, and perception of feedback messages, what relationship? In: Revista GalegoPortuguesa de Psicoloxía e Educación. Vol. 18, (1), Ano 14-2010, p. 133 - 146. 
León, J. M. R.; Delgado, T. G. (1998). Atribución causal. In: LEÓN e cols. Psicología Social: orientaciones teóricas y ejerciciso prático, Madrid : MacGrwhill.

Mascarenhas, S. A. N. (2004). Avaliação dos enfoques de aprendizagem dos alunos do ensino médio do estado de Rondônia, Tese doutoral, Universidade de La Coruña, Espanha, 822p. (inédita).

Mascarenhas, S. (2004). Atribuições causais $e$ rendimento no ensino médio. Rio de Janeiro: Ed. Autora.. ISBN 85-904846-2-9.

Mascarenhas, S, S.; Almeida, L.; Barca, A. (2005). Atribuições causais e rendimento escolar: impacto das habilitações escolares dos pais e do gênero dos alunos. In: Rev. Portuguesa de Educação, ano/vol. 18, número 001, Universidade do Minho, Braga: Portugal, p.77-91. 2005. Disponível em: $<$ http://repositorium.sdum.uminho. pt/handle/1822/29>. Recuperado em 10 abril 2010.

Mascarenhas, S. A. do N., Barca, A., L.,Silva, I. R. da, Maciel, A. C. \& Silva, G. C. R. F. da( 2013). Enfoques/Abordagens de Aprendizagem, atribuições causais, metas para estudar e rendimento acadêmico de estudantes do ensino superior, pág.29-45. In. Mascarenhas, S. A. do N. (Coord.) Determinantes do Rendimento Acadêmico no Ensino Superior, São Paulo, Loyola

Mascarenhas, S. A. do N., Boruchovitch, E, Maciel, A. C. \& Silva A. Q. da. (2013). Motivação para aprender e rendimento acadêmico de universitários, pág.93-101. In. Mascarenhas, S. A. do N. (Coord.) Determinantes do Rendimento Acadêmico no Ensino Superior, São Paulo, Loyola

Mascarenhas, S. A. do N., Silva, G. C. R da, Silva, I. R. da, Morais, L. M. de, Aguiar, R. da S., Peluso, M. L. A \& Barca, A. L. (2013). Atribuições causais e rendimento na universidade, pág.30-53. In. Mascarenhas, S. A. do N. (Coord.) Determinantes do Rendimento e do Bem Estar Psicossocial em Contextos Educativos Formais, São Paulo, Loyola.

Mascarenhas, S. A. do N \& Morais, L. M. de (2013). Motivação para aprender e rendimento acadêmico no ensino superior, pág.70-81. In. Mascarenhas, S. A. do N. (Coord.) Determinantes do Rendimento e do Bem Estar Psicossocial em Contextos Educativos Formais, São Paulo, Loyola.

Miras, M. (2004). Afetos, emoções, atribuições e expectativas: o sentido da aprendizagem escolar. In: CÓLL, C. \& Cols. Desenvolvimento psicológico e educação - Psicologia da educação escolar, vol. 2. Porto Alegre: Artmed, p.209-222.

Morais, L. M.; Mascarenhas, S. A. N. (2012). Avaliação dos efeitos dos enfoques de aprendizagem e da motivação para aprender sobre o rendimento acadêmico de estudantes da UFAM. Relatório técnico parcial de iniciação científica-PIBH006/UFAM/CNPq. Humaitá-AM.

Oliveira, A. M. B. de (1996). Atribuições causais e expectativas de controlo do desempenho na matemática, Universidade do Minho, IEP-CEEP, Braga, Portugal.

Peluso, M. L. A.; Mascarenhas, S. A. N.(2011). Avaliação dos enfoques de aprendizagem, atribuições causais, representações sociais, relações família - universidade, bem-estar subjetivo e rendimento de universitários do Amazonas/UFAM. Relatório técnico de iniciação científica-PIB-H034/UFAM/CNPq. HumaitáAM.

Rodrigues, A.(1984). Atribuição de causalidade: estudos brasileiros. In: Arquivos Brasileiros de Psicologia. Rio de Janeiro: Fundação Getúlio Vargas, v. 36, n.2, p. 5-20.

Sales, K. F. S.(2010) As atribuições causais de professores por fracasso escolar em matemática: comparação com percepções de seus alunos. Dissertação (Mestrado em Educação) Universidade Estadual de Londrina, Centro de Educação, Comunicação e Artes, Programa de Pós-Graduação em Educação, 85f.

Silva, G. C. R. F.; Mascarenhas, S. A. N.; Silva, I. R. (2011). O aporte da teoria das atribuições causais para a compreensão da afetividade na aprendizagem. In: Semana Educa, vol. 1, $\mathrm{n}^{\mathrm{o}} 1$, 2010. Disponível em: $<$ http://www.periodicos.unir.br/index.php/semana educa/index $>$. Recuperado em: 20 janeiro 2011.

Silva, G. C. R; Mascarenhas, S. A. N; Silva, I. R. (2011). Vivências de reprovação e as atribuições causais de estudantes sobre o rendimento escolar em Manaus. In: Anais do Congresso Nacional de Psicologia Escolar e Educacional: Universidade Estadual de Maringá - PR.

Weiner, B. (1985). An attributional theory of achievement motivation and emotion. In: Psychology Review, v. 92, n. 4, p. 548-73.

Weiner, B. (2004) Atribuition Theory revisited : transforming cultural plurality into theoretical unity. In: Research on sociocultural influences on motivation and learning. In: Big Teories Revisited, vol 4, p. 13-29. 